\title{
ERBB receptors in cancer: signaling from the inside
}

Carlos L Arteaga $a^{1,2,3 *}$

\begin{abstract}
ERBB receptor tyrosine kinases are activated by ligand-induced dimerization followed by activation and transphosphorylation of their intracellular kinase domains. A recent study by Bill and colleagues demonstrates that receptor transphosphorylation can be regulated from inside the cell by members of the cytohesin protein family. These data highlight a novel mechanism of amplification of ERBB receptor signaling output that may contribute to embryogenesis and cancer progression.
\end{abstract}

The ERBB receptor family encompasses four transmembrane tyrosine kinases that regulate cell differentiation, mitogenesis, survival, and migration. Dysregulated function of these receptor tyrosine kinases has been shown to result in cell transformation and cancer [1]. This receptor family includes the epidermal growth factor receptor (EGFR/ERBB1), HER2/ERBB2, HER3/ERBB3, and HER4/ ERBB4. Signaling is triggered by ligand binding to the extracellular domain of EGFR, ERBB3, and ERBB4, resulting in a conformational change in the receptor ectodomains and the dimerization of their cytoplasmic tyrosine kinase domains [2]. The receptor-receptor association releases the kinase domain from a default autoinhibited state, leading to transphosphorylation of tyrosine residues in the receptor C-terminus [3]. In turn, these phosphorylated tyrosines become docking sites for the specific binding of cytoplasmic signaling proteins containing Src homology-2 and protein tyrosine-binding domains, which then trigger several intracellular signaling pathways associated with cell growth and transformation [4].

Dimerization and activation of the tyrosine kinase domain of ERBB receptors are highly regulated processes

\footnotetext{
*Correspondence: carlos.arteaga@vanderbilt.edu

'Department of Medicine, Vanderbilt-Ingram Cancer Center, Vanderbilt University School of Medicine, Division of Hematology/Oncology, VUMC, 2220 Pierce Avenue, 777 PRB, Nashville, TN 37232-6307, USA

Full list of author information is available at the end of the article
}

modulated by incompletely understood stimulatory and inhibitory inputs. Recent data suggest that the mere dimerization of EGFR is not sufficient for their activation [5]. Further, only a fraction of dimerized receptors appears catalytically active, particularly those receptors where the kinase domains are arranged as asymmetric dimers [6,7]. These data coupled with the observation that receptor dimers may occur in the absence of ligand [5] led to speculation on the presence of cytoplasmic activators that modulate the conversion of these receptor dimers into an active state.

The study by Bill and colleagues now reports that cytohesins - guanine-nucleotide exchange factors of the ATP ribosylation factors involved in vesicular trafficking, cell migration, and cytoskeletal dynamics - facilitate conformational rearrangements in the cytoplasmic domain of ERBB receptors, leading to their activation [8]. The cytohesin family consists of four highly homologous proteins, the ubiquitous cytohesin-1, cytohesin-2 (ARNO), cytohesin-3 (Grp), and cytohesin-4, exclusively expressed by immune cells [9]. In this paper, overexpression of ARNO increased EGFR phosphorylation and this effect was also seen with a guanine-nucleotide exchange factor-inactive ARNO mutant. Receptor kinase activation was not associated with nor required receptor dimerization or endocytosis, suggesting a possible role for ARNO in asymmetric dimer formation. Using fluorescence resonance energy transfer and fluorescently labeled C-terminal EGFR fragments, Bill and colleagues showed that ARNO modulates the interaction between the two receptors in the dimer. Subsequent experiments suggested ARNO binds dimerized EGFR at the kinase or juxtamembrane regions. Similar effects were observed on endogenous, already dimerized ERBB2/ERBB3 receptors upon overexpression of ARNO into SKBR3 cells.

Tissue levels of ARNO were evaluated by immunohistochemistry in a cohort of primary lung adenocarcinomas. Staining with ARNO antibodies appeared higher in the cancers compared with normal lung tissues. Further, tissue levels of the cytohesin correlated with levels of P-EGFR, P-ERK, and P-AKT also measured by immunohistochemistry. Inhibition of ARNO with the cytohesin inhibitor SecinH3 or siRNA reduced basal EGFR phosphorylation/activation and growth of EGFRdependent human lung adenocarcinoma cells in vitro. 
Further, administration of SecinH3 to nude mice bearing PC9 lung cancer xenografts inhibited tumor cell proliferation measured by $\left[{ }^{18} \mathrm{~F}\right]$-fluoro-L-thymidine uptake positron emission tomography and Ki67 immunohistochemistry as well as inducing tumor cell apoptosis assessed by terminal deoxynucleotidyl transferase dUTP nick endlabeling analysis. PC9 cells harbor a gain-of-function activating mutant of EGFR [10], which suggests the facilitating role of ARNO on ERBB signaling is not limited to wild-type receptors.

These studies have important implications for cancer biology and therapy as they identify a novel mechanism usurped by transformed cells to amplify ERBB receptor signaling from the cytoplasmic side, and thus increase the number of actively signaling ERBB dimers within the pool of ligand-occupied receptors. As such, overexpression of cytohesins would provide a gain-of-fitness to ERBB signaling output that can be utilized by cancer cells in stressful situations such as fragile windows during metastatic progression and/or when they are exposed to anti-cancer therapies. This role of cytohesins may extend to heterologous receptors as suggested by the fact that SecinH3 reduced phosphorylation of IRS-1, a main signal transducer of insulin and IGF-I receptor tyrosine kinases, in H460 lung cancer cells [8]. Future studies should also address whether ARNO overexpression occurs over a wider range of ERBB-dependent cancers. The experiments with ERBB2 gene-amplified and ERBB2-dependent SKBR3 cells suggest that cytohesins can also fine tune signaling by (asymmetric) ERBB2/ERBB3 dimers, where the potent ERBB2 kinase activates the catalytically deficient kinase domain of ERBB3. These heterodimers are the most potent signaling complexes of the ERBB family [4] and are critical for the viability of breast cancer cells with $E R B B 2$ gene amplification [11,12]. Whether overexpression of cytohesins, by amplifying ERBB signaling, dampens the response to tyrosine kinase inhibitors or receptor antibodies approved for the treatment of ERBBdependent tumors remains to be studied.

In summary, the elegant paper by Bill and colleagues shows that cytohesins promote ERBB receptor signaling by facilitating the formation of asymmetric dimers. For those interested in translational research, these data introduce other assays that can be incorporated into metrics aimed at measuring ERBB receptor output in tumor tissues in situ, such as the tumor content of cytohesins. Finally, the data suggest inside activators of ERBB receptor signal transduction as therapeutic targets that may also need to be interrupted in order to have a maximal effect for currently approved anti-ERBB receptor therapies.
Abbreviations

EGFR, epidermal growth factor receptor; siRNA, small interfering RNA.

\section{Competing interests}

The author declares that he has no competing interests.

\section{Acknowledgements}

The present work as supported by NCI R01 Grant CA80195, ACS Clinical Research Professorship Grant CRP-07-234, the Lee Jeans Translational Breast Cancer Research Program, Breast Cancer Specialized Program of Research Excellence (SPORE) Grant P50 CA98131, and Vanderbilt-Ingram Cancer Center Support Grant P30 CA68485.

\section{Author details}

'Department of Medicine, Vanderbilt-Ingram Cancer Center, Vanderbilt University School of Medicine, Division of Hematology/Oncology, VUMC, 2220 Pierce Avenue, 777 PRB, Nashville, TN 37232-6307, USA. ${ }^{2}$ Department of Cancer Biology, Vanderbilt-Ingram Cancer Center, Vanderbilt University School of Medicine, Division of Hematology/Oncology, VUMC, 2220 Pierce Avenue, 777 PRB, Nashville, TN 37232-6307, USA. 'Breast Cancer Research Program, Vanderbilt-Ingram Cancer Center, Vanderbilt University School of Medicine, Division of Hematology/Oncology, VUMC, 2220 Pierce Avenue, 777 PRB, Nashville, TN 37232-6307, USA.

Published: 16 March 2011

\section{References}

1. Bublil EM, Yarden Y: The EGF receptor family: spearheading a merger of signaling and therapeutics. Curr Opin Cell Biol 2007, 19:124-134.

2. Yarden Y, Schlessinger J: Self-phosphorylation of epidermal growth factor receptor: evidence for a model of intermolecular allosteric activation. Biochemistry 1987, 26:1434-1442.

3. Ferguson KM: Structure-based view of epidermal growth factor receptor regulation. Annu Rev Biophys 2008, 37:353-373.

4. Yarden Y, Sliwkowski MX: Untangling the ErbB signalling network. Nat Rev Mol Cell Biol 2001, 2:127-137.

5. Chung I, Akita R, Vandlen R, Toomre D, Schlessinger J, Mellman I: Spatial control of EGF receptor activation by reversible dimerization on living cells. Nature 2010, 464:783-787.

6. Zhang X, Gureasko J, Shen K, Cole PA, Kuriyan J: An allosteric mechanism for activation of the kinase domain of epidermal growth factor receptor. Cell 2006, 125:1137-1149.

7. Jura N, Endres NF, Engel K, Deindl S, Das R, Lamers MH, Wemmer DE, Zhang X, Kuriyan J: Mechanism for activation of the EGF receptor catalytic domain by the juxtamembrane segment. Cell 2009, 137:1293-1307.

8. Bill A, Schmitz A, Albertoni B, Song JN, Heukamp LC, Walrafen D, Thorwirth F, Verveer PJ, Zimmer S, Meffert L, Schreiber A, Chatterjee S, Thomas RK, Ullrich RT, Lang T, Famulok M: Cytohesins are cytoplasmic ErbB receptor activators. Cell 2010, 143:201-211.

9. Kolanus W: Guanine nucleotide exchange factors of the cytohesin family and their roles in signal transduction. Immunol Rev 2007, 218:102-113.

10. Sharma SV, Bell DW, Settleman J, Haber DA: Epidermal growth factor receptor mutations in lung cancer. Nat Rev Cancer 2007, 7:169-181.

11. Holbro T, Beerli RR, Maurer F, Koziczak M, Barbas CF, 3rd, Hynes NE: The ErbB2/ErbB3 heterodimer functions as an oncogenic unit: ErbB2 requires ErbB3 to drive breast tumor cell proliferation. Proc Natl Acad Sci U S A 2003 100:8933-8938.

12. Lee-Hoeflich ST, Crocker L, Yao E, Pham T, Munroe X, Hoeflich KP, Sliwkowski MX, Stern HM: A central role for HER3 in HER2-amplified breast cancer: implications for targeted therapy. Cancer Res 2008, 68:5878-5887.

doi:10.1186/bcr2829

Cite this article as: Arteaga $C L$ : ERBB receptors in cancer: signaling from the inside. Breast Cancer Research 2011, 13:304. 